\title{
Effectiveness of Modified Constraint Induced Movement Therapy and Bilateral Arm Training on Upper Extremity Function after Chronic Stroke: A Comparative Study
}

\author{
Damayanti Sethy ${ }^{1 *}$, Pankaj Bajpai ${ }^{1}$, Eva Snehlata Kujur ${ }^{1}$, Kshanaprava Mohakud ${ }^{2}$, \\ Surjeet $\mathrm{Sahoo}^{3}$ \\ ${ }^{1}$ Department of Occupational Therapy, $\mathrm{NIOH}$, Kolkata, West Bengal, India \\ ${ }^{2}$ SVNIRTAR, Odisha, India \\ ${ }^{3}$ Siksha 'O' Anusandhan University, Odisha, India \\ Email: "damayanti.sethy@gmail.com,pankajda@yahoo.com, eskujur@yahoo.com \\ Id-kshanapravamohakud@yahoo.com, surjeetsahoo@gmail.com
}

Received 2 November 2015; accepted 12 February 2016; published 15 February 2016

Copyright (C) 2016 by authors and Scientific Research Publishing Inc.

This work is licensed under the Creative Commons Attribution International License (CC BY). http://creativecommons.org/licenses/by/4.0/

(c) (i) Open Access

\section{Abstract}

Statement of the Problem: Upper limb hemiparesis is a common impairment underlying disability after Stroke. Transfer of treatment to daily functioning remains a question for traditional approaches used in treatment of upper extremity hemiparesis. Approaches based on Motor Learning principles may facilitate the transfer of treatment to activities of daily living. Methodology: Forty one subjects with chronic stroke, attending department of occupational therapy, National Institute for the Orthopaedically Handicapped, Kolkata, West Bengal, India participated in a single blinded randomized pre-test and post-test control group training study. Subjects were randomized over three intervention groups receiving modified Constraint Induced Movement Therapy $(n=13)$, Bilateral Arm training $(n=14)$, and an equally intensive conventional treatment program $(n=14)$. Subjects in the bilateral arm training group participated in bilateral symmetrical activities, where as subjects in constraint induced movement therapy group performed functional activities with the affected arm only and conventional group received conventional Occupational Therapy. Each group received intensive training for 1 hour/day, 5 days/week, for 8 weeks. Pre-treatment and post-treatment measures included the Fugl-Meyer measurement of physical performance (FMAupper extremity section), action research arm test, motor activity log. Assessments were administered by a rater blinded to group assignment. Result: Both $m$-CIMT $(p=0.01)$ and bilateral arm training $(p=0.01)$ group showed statistically significant improvement in upper extremity func-

\footnotetext{
${ }^{*}$ Corresponding author.
} 
tioning on Action Research Arm Test score in comparison to the conventional therapy group ( $p=$ 0.33). The bilateral arm training group had significantly greater improvement in upper arm function (Proximal Fugl-Meyer Assessment score, $p=0.001$ ); while the constraint induced movement therapy group had greater improvement of hand functions (Distal Fugl-Meyer Assessment score, $p$ $=0.001$. There is an improvement seen in Quality of movement in the Conventional Therapy group. $(p=0.001)$. Conclusion: Both the treatment techniques can be used for upper extremity management in patients with chronic stroke. Bilateral arm training may be used to improve upper arm function and m-CIMT may be used to improve hand functions, while the group that received modified constraint induced movement therapy had greater improvement.

\section{Keywords} Stroke, Rehabilitation, Upper Extremity, Bilateral Arm Training, Constraint Induced Movement
Therapy

\section{Introduction}

\subsection{Relevance to Occupational Therapy Field}

Stroke is the third leading cause of death and the leading cause of adult disability [1]. Upper limb hemi paresis is a common impairment underlying disability after CVA. Dysfunction from upper extremity hemi paresis impairs performance of many daily activities such as dressing, bathing, self care and writing, thus reducing functional independence. In fact only $5 \%$ of adults regain full arm function after stroke, and $20 \%$ regain no functional use [2]. Restoration of full function to the stroke affected upper extremity is a major problem in rehabilitation. Hence alternative strategies are needed to reduce the long-term disabilities and functional impairment resulting from upper extremity hemi paresis [1].

\subsection{Literature Review}

Various conventional treatment technique are used for upper extremity management in patients with stroke using the principles of Bobath's Neuro-developmental Therapy, Brunnstorm's Movement Therapy, Rood's Techniques and Proprioceptive Neuromuscular Facilitation developed by Knott and Voss [3]. There is some question as to the efficacy of these approaches with regard to transfer of treatment effect in life situation [4]. Only one third of all stroke patients regain some dexterity within six months using conventional treatment programs [5]. In light of the above fact, some recent studies based on the principle of neuroplasticity, have produced promising results in improving upper extremity functioning in patients with chronic stroke. One such intervention is Constraint induced movement therapy (CIMT). In CIMT, the affected upper extremity is trained intensively by constraining the non paretic arm. This is based on the principle of forced use and task specificity. This intensive practice induces use dependent cortical reorganization; there by increasing the use of affected arm [6]. Various randomized controlled trials have showed CIMT to be an effective method for improving upper extremity function in patients with chronic stroke [2] [7] [8].

In contrast, bilateral arm training with rhythmic auditory cueing (BATRAC) has been shown to have beneficial effects on upper extremity function in patients with chronic stroke [1]. In bilateral arm training, the principle of forced use and task specificity was retained, without constraining the non paretic arm and both the arms are trained. Although neural mechanisms remain unclear, it has been speculated that bilateral practice may facilitate co activation and inter hemispheric activation, and thus a possible change in the contralesional cortical network [9]. The above facts suggest that training may also induce beneficial changes in the contralesional brain area, rather than only in ipsilesional brain area [9]. This raises a question whether unilateral training or bilateral training is better for improving upper extremity function in patients with chronic stroke in comparison to conventional treatment program.

In this study an attempt has been made to investigate the effectiveness of all three intervention programs in patients with chronic stroke and to compare which of the three interventions, is more effective in terms of recovery of upper extremity function in patients with chronic stroke. 
Here the protocol of bilateral arm training (BAT) is based on BATRAC, but auditory cueing was excluded and a custom made bilateral training equipment was used. As there are various forms of CIMT are available, in this study modified constraint induced movement therapy (m CIMT) was used. Conventional Occupational Therapy was based on techniques of Bobath, Brunnstrom, Rood's and Proprioceptive Neuromuscular Facilitation (PNF).

\subsection{AIM of the Study/Purpose}

1) Investigating the effectiveness of two intervention programs based on Motor Learning approach and one intervention based on Conventional Occupational Therapy approach.

2) Comparing all three approaches for improving upper extremity function in chronic stroke.

\section{Research Methodology}

\subsection{Design}

This study was a single blind, randomized pre test and post test control group design. Forty one patients consisting of 24 men and 17 women with chronic hemi paretic arm dysfunction were recruited from outpatient Occupational Therapy Department and indoor rehabilitation ward of National Institute for the Orthopaedically Handicapped (NIOH). Informed consent was obtained from all the patients before inclusion in the study. Inclusion criteria were as follows; at least 6 months since a unilateral stroke, ability to follow simple instructions and two step commands (Mini mental state score > 22) [10], ability to actively extend at least 10* at metacarpophallangeal joint and interphallangeal joints and 20* at the wrist joint, age between 30 and 75 years. Exclusion criteria were uncontrolled hypertension (190/110 mm Hg), significant orthopeadic and pain conditions, excessive spasticity, defined as a score of 3 or more on Modified As worth scale (MAS) [3]. The patients were randomly allocated to Group-A (m-CIMT), Group-B (BAT) and Group-C (Conventional Therapy) by writing the group names with a number on paper slips. All the patients were asked to draw one paper slip and according to the numbers assigned to each group, patients were allocated to the respective groups. Group-A (modified Constraint induced movement therapy) consisted of 13 of 08 men and 5 women patients with chronic stroke and Group-B (Bilateral arm training) consisted of 14 of 07 men and 07 women and Group-C (conventional therapy) consisted of 14 of 9 men and 5 women patients with chronic stroke. The assessor was blinded to the participant groups.

\subsection{Outcome Measures}

- Fugl-Meyer measurement of physical performance-Upper Extremity Section (FMA)-FMA is a 66-point, upper extremity section of FMA [11], which assesses several impairment dimensions by using a 3-point ordinal scale (0, cannot perform: 1, can perform partially; 2, can perform fully). The FMA has impressive test retest reliability (total range 0.98 - 0.99; subtests range, 0.87 - 1.00), Inter rater reliability, and construct validity [11].

- Action Research Arm test (ARAT): ARAT is an observational scale used to measure upper extremity function. The ARA Test is a 19 item test divided into 4 categories (grasp, grip, pinch, gross movement), with each item graded on a 4 point ordinal scale ( 0 , can perform no part of the test; 1 , perform test partially; 2 , completes test but takes abnormally long time or has great difficulty; 3, performs test normally) for a total possible score of 57 [12]. The test is hierarchical in that, if the patient can perform each category, he or she will be able to perform the other items within the category and, thus, they need not be tested. The ARA has high inter rater reliability $(r=0.99)$ and test retest reliability $(r=0.98)$, and it has high validity [12].

- Motor Activity Log (MAL): The MAL is a semi structured interview, where the patient and their care givers independently rate how much and how well the patient has used the affected arm for 30 ADL activities in the past week. Patients and care givers use a 6-point AOU (Amount of use) scale to rate how much they are using their affected arm and 6-point scale to rate QOU (Quality of use) to rate how well the arm is being used. A higher score indicates better performance. The MAL has established good test-retest reliability, internal consistency, stability and responsiveness and convergent validity [13].

\subsection{Procedure}

The stroke patients who fit the inclusion criteria were allotted to three groups after getting the informed consent. 
The study was approved by the Ethics committee of the Institute. A general history including score on Mini Mental State Examination (MMSE), Modified As worth Scale was taken from the patient, after which FMA and ARA, and MAL were administered by an Occupational Therapist independent of the group assignment. MMSE was used only for the screening purpose. m-CIMT was given to group-A and bilateral arm training was given to Group-B and conventional therapy was given to Group-C. Patients in all three groups received therapy for one hour per day, 5 days/week for 8 weeks.

\subsection{Interventions}

\subsubsection{Modified Constraint Induced Movement Therapy}

Modified Constraint Induced Movement Therapy aimed at improving motor task performance of the paretic arm and hand. Modified CIMT comprised of the following components

\section{First component}

The patients in this group participated in one hour session of occupational therapy, 5 days/week for 8 weeks.

During first component patients performed one hour of activity session and a rest period of 5 minutes was given between each 10 minutes of task practice. The activities include some of the basic activities of daily living and instrumental activities of daily living. The activities were:

- Feeding - consistency of the food will vary from solid to semisolid;

- Drinking-holding the glass and taking it to mouth;

- Simulated bathing, Combing hair;

- Manipulating switches, taps, latches, writing, using telephones, playing activities like Plastic peg activity, stacking blocks, setting bed and table.

The involved upper extremity was facilitated and trained in the above tasks through a process called "shaping”. This is done by a technique called successive approximation. The tasks were broken down into smallest steps [4] [14].

This process involved presenting unimanual interesting and useful activities that provide immediate feedback for the patient's effort and increasingly functional use of more impaired upper extremity. The content and the duration of training were recorded in a logbook for each patient.

\section{The second component of m-CIMT}

Unaffected upper extremity of the patient was constrained by a cotton glove, which covered the hand a little above the wrist. The arm was secured using a cotton sling, which was then fastened around the neck. Patient was advised to use the restraint for 5 hours that were considered as the time of frequent use and asked to keep a record of the time period worn.

\subsubsection{Bilateral Arm Training}

Patients were seated comfortably in a chair in front of a table. Bilateral arm trainer was fixated on a table and was designed by using wooden material. The trainer has got an overhead rack for placement of blocks and pegs. The rack was fitted to two parallel wooden bar. Both the bars are fixated to both sides of the table and the rack was fitted horizontally over the parallel bars. Velcro hooks were attached to both the upper and under surface of the overhead rack where the patient can place the blocks and pegs. The height of the over head rack was adjustable. Nuts and screws were fitted in the side parallel bar in order to change the height of the rack. Blocks and pegs were also attached with Velcro loops.

Each session involve repetitive practice of bilateral tasks for one hour. The tasks are:

- Block placement-10 minutes - the patient has to pick up one block from the container and transport it to the over head rack and place it above it. The same will be done bilaterally for 15 repetitions.

- Peg targeting -10 minutes - the patient has to grasp the pegs using both the hands and transport it to the targets on the rack where Velcro is attached.

- Peg inversion-10 minutes - two pegs has to be picked up by using both the hands, rotated using supination and then placed over the under surface of the rack.

- Transferring object from one container to another-10 minutes.

Rest periods of 5 minutes each at four intervals between the tasks were given to each patient to reduce the effect of conditioning. Same tasks were maintained for each patient over the duration of the study. Practice was done using bilateral upper extremity simultaneously. The common movements in the bilateral task training are shoulder flexion/protraction and elbow extension, and shoulder extension/retraction and elbow flexion. This ac- 
tion mimics the behavior of reaching for an object and bringing it to oneself.

\subsubsection{Conventional Occupational Therapy}

Conventional Occupational Therapy included techniques based on Bobath Neuro Developmental Therapy approach. Weight bearing for upper limb, Reflex Inhibiting patterns (RIPs), Trunk rotation and scapular protraction were used to reduce spasticity. This was followed by activities.

Post training all the three groups were measured for FMA, ARA, and MAL.

\section{Statistical Analysis}

SPSS version 17.0 was used for all the statistical analysis. The initial analyses were 1-way ANOVA to compare the pre test and post test measures on the dependent variables. Significant results were further investigated with post hoc (Tukey honestly significant difference) comparisons and a Bonferroni correction was used.

\section{Results}

All the three groups in the study were compared both pre test and post test. Homogeneity of the subsets was tested for all the outcome measures used. The characteristics of the subjects are presented in Table 1. Baseline patient characteristics were comparable across these three groups $(\mathrm{p}=0.988)$.

The ARA Test scores showed significant improvement on post test $(\mathrm{p}=0.01)$. Post hoc analysis revealed that the post test score was significantly higher than the pre test score in both BAT and m-CIMT group. The conventional group had nominal improvement on ARA scores $(\mathrm{p}=0.33)$. The graphical representation is given in Figure 1 .

The Fugl-Meyer Upper extremity motor performance section Test scores showed significant improvements in Bilateral arm training group $(p=0.001)$ and Modified CIMT group $(p=0.011)$. Subjects in BAT group and $\mathrm{m}$-CIMT group exhibited greater gains than did conventional therapy subjects $(\mathrm{p}=0.693)$. There was no significant difference between BAT and m-CIMT group, except BAT group showed better performance in the proximal arm score of FMA. Post hoc analysis revealed that the post test score was higher than the pre test score $(\mathrm{p}=$ $0.001)$. m-CIMT group showed better performance in the distal arm score. $(\mathrm{p}=0.001)$ The graphical representation is given in Figure 2.

The MAL test scores showed significant improvement on post test $(\mathrm{p}=0.00)$. Post hoc analysis revealed that the post test score of Amount of use (AOU) $(p=0.001)$ and Quality of movement $(\mathrm{QOM})(\mathrm{p}=0.000)$ are significantly higher than the pre test score both in BAT and m-CIMT group. The graphical representation is given

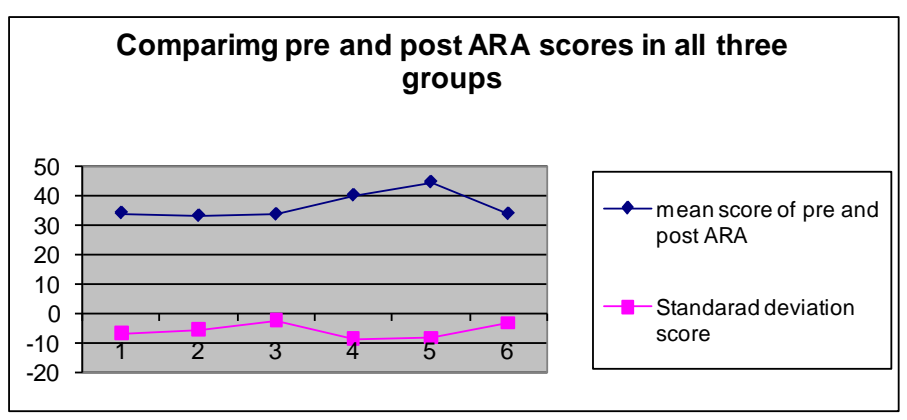

Figure 1. Comparision of pre post ARA scores across all three groups.

Table 1. Characteristics of subject population.

\begin{tabular}{|c|c|c|c|c|c|}
\hline Sl no & Characteristics & $\begin{array}{l}\text { Modified-constraint induced movement } \\
\text { therapy (m-CIMT) group mean(S.D) }\end{array}$ & $\begin{array}{l}\text { Bilateral arm training } \\
\text { (BAT) group mean (S.D) }\end{array}$ & $\begin{array}{l}\text { Conventional therapy } \\
\text { mean (S.D) }\end{array}$ & Significance \\
\hline 1 & No of subjects & 13 & 14 & 14 & \\
\hline 2 & Age (in years) & $58.11(9.30)$ & $57.34(11.92)$ & $57.59(11.03)$ & 0.686 \\
\hline 3 & Sex (male/female) & $9 / 5$ & $10 / 04$ & 09/05 & 0.892 \\
\hline 4 & $\begin{array}{l}\text { Side of lesion, No } \\
\text { left/right. }\end{array}$ & 08/06 & 09/05 & $10 / 04$ & 0.660 \\
\hline 5 & Months since stroke & $12.35(3.21)$ & $13.09(2.86)$ & $13.82(3.01)$ & 0.812 \\
\hline
\end{tabular}




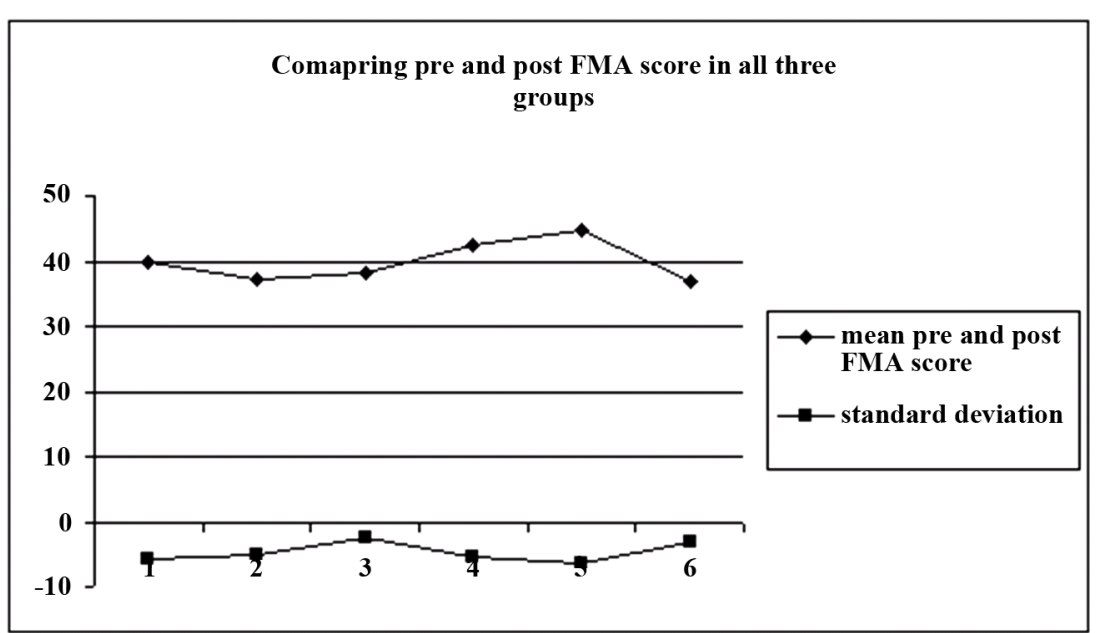

Figure 2. Comparision of pre post FMA scores across all three groups.

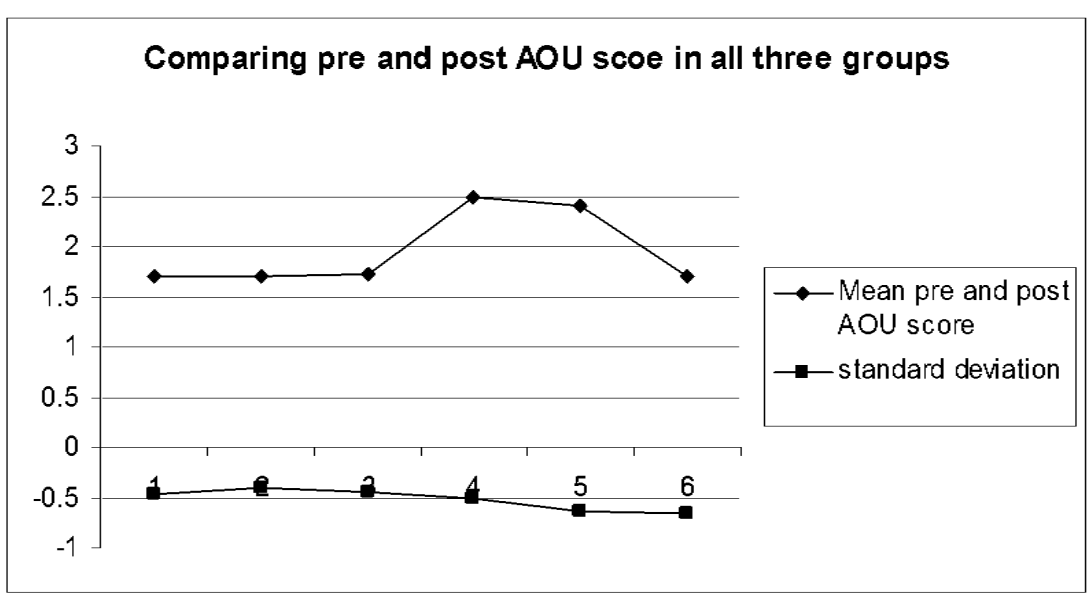

Figure 3. Comparision of pre post AOU scores across all three groups.

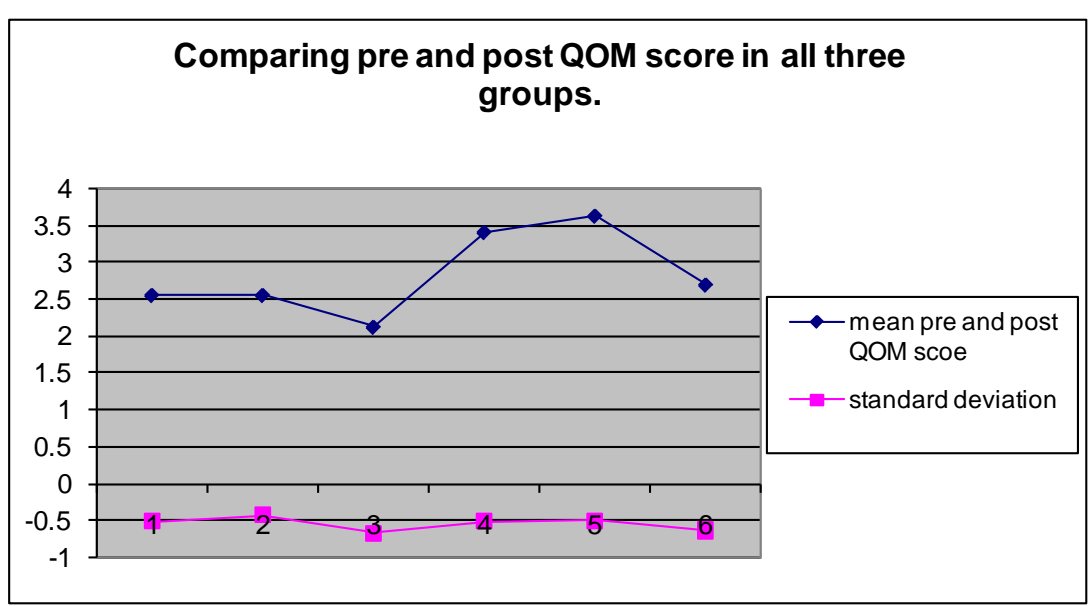

Figure 4. Comparision of pre post QOM scores across all three groups.

in Figure 3 and Figure 4. Both these groups showed greater improvements than conventional therapy group in the amount of use and quality of movement of the affected arm during performance of daily activities. Though there was no significant improvement in the amount of use in conventional Occupational Therapy group, but 
Table 2. Descriptive and inferential statistics for the outcome measures.

\begin{tabular}{cccccccccc}
\hline \multirow{2}{*}{ Outcome measure } & \multicolumn{7}{c}{ Pre treatment } & \multicolumn{3}{c}{ Post treatment } & f \\
\cline { 2 - 7 } & m-CIMT & BAT & CT & m-CIMT & BAT & CT & \\
ARA & 34.07 & 33.14 & 33.60 & 40.00 & 44.4 & 33.78 & 8.219 & 0.001 \\
mean (S.D) & $(6.89)$ & $(5.60)$ & $(2.34)$ & $(8.77)$ & $(8.29)$ & $(3.28)$ & & \\
FMA & 39.78 & 37.28 & 38.28 & 42.35 & 44.78 & 36.92 & 8.444 & 0.001 \\
mean (S.D) & $(5.82)$ & $(5.01)$ & $(2.33)$ & $(5.55)$ & $(6.26)$ & $(3.22)$ & & \\
MAL & 1.71 & 1.71 & 1.73 & 2.5 & 2.4 & 1.7 & 11.777 & 0.000 \\
AOU mean (S.D) & $(0.46)$ & $(0.41)$ & $(0.44)$ & $(0.51)$ & $(0.64)$ & $(0.65)$ & \\
QOM mean (S.D) & 2.57 & 2.57 & 2.14 & 3.42 & 3.64 & 2.71 & 45.190 & 0.000 \\
\hline
\end{tabular}

Ara—action research arm test, fma-Fugl-Meyer assessment of upper extremity function, mal—motor activity log, aou—amount of use, qom—quality of movement.

there was a significant improvement in quality of movement in this group $(p=0.001)$. This shows that conventional Occupational Therapy is effective in improving the movement quality of upper extremity in chronic stroke patients. The descriptive and inferential statistics are given in Table 2.

\section{Discussion}

The result of the study provides experimental data addressing the changes that occurred in both motor and functional performance and quality of movement in patients with stroke following participation in a 8 week Bilateral Arm Training and modified Constraint Induced Movement Therapy relative to conventional Therapy. The motor Activity Log scores showed greater improvements in quality of movement and amount of use in BAT and m-CIMT group in comparison to Conventional Therapy.

In this study we found, 8 weeks of BAT showed improvement in measures of motor impairment and functional use in patients with chronic upper extremity hemiparesis. This suggests that forced use in a repetitive manner, bilaterally can improve motor ability and functional use of upper extremity in chronic hemiparetic stroke patients. This result is consistent with the findings of Whittal et al., 2000 and Luft et al., 2004. In our study the BAT group showed better performance in the proximal part score of FMA than m-CIMT and Conventional Therapy group. The repetitive reaching type movements comprising shoulder flexion, protraction, elbow extension and shoulder extension/retraction; elbow flexion might have resulted in the improvement of proximal part motor ability, thereby improving the proximal part score of FMA in bilateral arm training group relative to m-CIMT and Conventional Therapy group. The effectiveness of the bilateral arm training can be found in the behavior and neurophysiology literature. Practicing bilateral simultaneous movements may result in a facilitation effect from non-paretic arm For example, when the bimanual movements are initiated simultaneously, the arms act as a unit that supersedes individual arm action, indicating that both arms are strongly linked as a coordinated unit in the brain, (the entrainment effect). Studies by Kelso J.A., Putnam C.A., Goodman D., 1979 [15] on inter limb coordination during simultaneous performance of bimanual tasks suggest that when both limbs are performing identical actions, the same movement organization occurs in both hemispheres. In fact there may be a single command or central mechanisms applied to both limbs. When the two hands perform identical tasks, there is a tight phasic relationship observed in which one limb entrains the other, causing them to function together as a unit. Another important aspect of bilateral arm training is repetition which is a well known motor learning principle and recent animal studies have demonstrated that forced use involving a motor task rather than forced use alone may best promote central nervous system plasticity [16].

The m-CIMT protocol used more intensive functional practice by the affected upper extremity. Intensive practice of the affected arm might provide sufficient proprioceptive and visual feed back to develop internal models for feed-forward control of movement [7] [8]. In m-CIMT group subjects showed greater gain in MAL, than did BAT and conventional therapy group. These findings are consistent with previous studies. MAL scores suggest that the functional use of the upper extremity improved better in m-CIMT group relative to BAT and conventional Therapy group. Modified CIMT group displayed changes greater than 2.5 on both AOU and QOM scales of the Motor Activity Log. Modified CIMT patients reported that they were capable of using the affected upper extremity more in Activities of daily living than previously used. This might have happened due to the 
more use of hand movements and manipulation of objects involved in functional tasks of m-CIMT group. Post training m-CIMT group showed considerable change in both FMA and ARA Test. The result of this study is supported by those of Levine P., Page S.J., 2004, Lewis G.N. et al., 2004 [17] who described that practice conveys motor skill learning, and m-CIMT allowed practice of the more affected arm and hand. Furthermore FMA and ARA data support the contention that m-CIMT participation can elicit functional changes.

In this study both BAT, m-CIMT group patients showed better improvement on FMA, ARA Test and MAL scores than conventional Therapy. This result is consistent with that of previous studies [17].

\section{Conclusion}

The result of the study demonstrated that both bilateral arm training and modified constraint induced movement therapy are efficacious for upper extremity hemiparesis in chronic stroke patients in comparison to conventional therapy. Bilateral arm training is more effective in improving proximal upper extremity functions and modified constraint induced movement therapy may improve functional use of the affected limb in chronic hemiparetic stroke patients. There were certain limitations in conducting the study. The study had a small sample size and no follow-up was done to see the maintenance effect of bilateral arm training and modified constraint induced movement therapy. Patients in the modified constraint induced movement therapy group received some encouragement while performance of the functional tasks that might have an impact on the result. The equipment used for bilateral arm training in the study was locally made without any electrical connection giving auditory or visual feedback for movement. The entire patient in the BAT group used their own speed for the movements. Further studies are necessary to assess the long term effects of BAT and m-CIMT. Since training was given only to chronic patients; future studies on acute and sub acute stroke population can be conducted. New designs of bilateral arm training involving wrist and hand can be incorporated and studied on different stroke population.

\section{References}

[1] Whittal, J., McCombe Waller, S., Silver, K.H.C., et al. (2000) Repetitive Bilateral Arm Training with Rhythmic Auditory Cueing Improves Motor Function in Chronic Stroke. Stroke, 31, 2390-2395. http://dx.doi.org/10.1161/01.STR.31.10.2390

[2] Bonifer, N.M., Anderson, K.M., Arciniegas, D.B., et al. (2005) Constraint Induced Movement Therapy for Stroke: Efficacy for Patients with Minimal Upper Extremity Motor Ability. Archives of Physical Medicine and Rehabilitation, 86, 1867-1872. http://dx.doi.org/10.1016/j.apmr.2005.04.002

[3] Radomski, M.V. and Trombly Latham, C.A. (2008) Occupational Therapy for Physical Dysfunction. 6th Edition, Lippincott Williams and Wilkins, Philadelphia.

[4] Taub, E., Uswatte, G. and Pidikiti, R. (1999) Constraint Induced Movement Therapy, a New Family of Techniques with Broad Application to Physical Rehabilitation-A Clinical Review. Journal of Rehabilitation Research and Development, 36, 273-251.

[5] Dobkin, B.H. (2005) Clinical Practice. Rehabilitation after Stroke. The New England Journal of Medicine, 352, 16771684. http://dx.doi.org/10.1056/NEJMcp043511

[6] Taub, E., et al. (1993) Techniques to Improve Chronic Motor Deficits after Stroke. Archive of Physical Medicine and Rehabilitation, 74, 347-354.

[7] Page, S.J., Levine, P., Sisto, S., et al. (2002) Stroke Patients and Therapists Opinions of Constraint Induced Movement Therapy. Clinical Rehabilitation, 16, 55-60. http://dx.doi.org/10.1191/0269215502cr473oa

[8] Page, S.J., Sisto, S., Levine, P. and McGrath, R.E. (2004) Efficacy of Modified Constraint Induced Movement Therapy in Chronic Stroke: A Single Blind Randomized Controlled Trial. Archives of Physical Medicine and Rehabilitation, 85, 14-17. http://dx.doi.org/10.1016/S0003-9993(03)00481-7

[9] Luft, A.R., McCombe-Waller, S., Whitall, J. et al. (2004) Repetitive Bilateral Arm Training and Motor Cortex Activation in Chronic Stroke. JAMA, 292, 1853-1861. http://dx.doi.org/10.1001/jama.292.15.1853

[10] Uswatte, G. and Taub, E. (1999) Constraint Induced Movement Therapy. New Approaches to Outcome Measurement in Rehabilitation. In: Struss, D.T., Winocur, G. and Robertson, I.H., Eds., Cognitive Neurorehabilitation, a Comprehensive Approach, Cambridge University Press, Cambridge, England, 215-29

[11] Fugl-Meyer, A.R., et al. (1975) The Post Stroke Hemiplegic Patient. I. A Method for Evaluation of Physical Performance. Scandinavian Journal of Rehabilitation Medicine, 7, 13-31.

[12] Vander Lee, J.H., Beckermen, H., Lankhorst, G.J. and Breter, L.M. (2001) The Responsiveness of the Action Research 
Arm Test and Fugl-Meyer Assessment of Physical Performance Scale in Chronic Stroke Patients. Journal of Rehabilitation Medicine, 33, 110-113. http://dx.doi.org/10.1080/165019701750165916

[13] Vander Lee, J.H., Wagenaar, R.C., Lankhorst, G.J., et al. (1999) Forced Use of the Upper Extremity in Chronic Stroke Patients: Results from a Single Blind Randomized Clinical Trial. Stroke, 30, 2369-2375. http://dx.doi.org/10.1161/01.STR.30.11.2369

[14] Staines, W.R., McIlroy, W.E., Graham, S.J. and Black, S.E. (2001) Bilateral Movement Enhances Ipsilesional Cortical Activity in Acute Stroke: A Pilot Functional MRI Study. Neurology, 56, 401-404. http://dx.doi.org/10.1212/WNL.56.3.401

[15] Kelso, J.A.S., Putnam, C.A. and Goodman, D. (1983) On the Space-Time Structure of Human Inter Limb Coordination. The Quarterly Journal of Experimental Psychology Section, 35A, 347-375. http://dx.doi.org/10.1080/14640748308402139

[16] Carr, J. and Shepherd, R. (1998) Neurological Rehabilitation: Optimizing Motor Performance. Butterworth-Heineman, Edinburgh, 241-264.

[17] Levine, P. and Page, S.J. (2004) Modified Constraint Induced Movement Therapy: A Promising Restorative out Patient Therapy. Top Stroke Rehabilitation, 11, 1-10. http://dx.doi.org/10.1310/R4HN-51MW-JFYK-2JAN 